\title{
La position du TFA relative à la transmission de rapports médicaux au médecin-conseil est juste dans sa conclusion mais incompréhensible dans ses motifs
}

Hanspeter Kuhn, avocat, secrétaire général adjoint
Dans son article, Christian Peter, dr en droit, résume l'arrêt du TFA et explicite les conséquences qui en résultent selon lui. Dans les lignes qui suivent, j'aimerais présenter les antécédents du cas, informer sur l'enseignement prodigué dans le cours de formation postgraduée en vue de l'attestation de formation complémentaire «médecinconseil» et finalement montrer pourquoi cet arrêt du TFA est, à mon avis, juste dans la conclusion mais incompréhensible dans les motifs.

\section{Le cas d'espèce}

Du 23 septembre 2002 au 12 novembre 2002, un «spécialiste FMH en médecine générale, médecin en gynécologie» a traité une patiente pour laquelle il a établi une facture s'élevant à Fr. 1052.20. Ce montant contient, outre la première période de traitement, également cinq rapports médicaux. Le suivi thérapeutique a lieu du 22 août 2003 au 6 novembre 2003, et cette période est facturée pour un montant de Fr. 1079.10.

Le 8 janvier 2004, le département des prestations de la caisse-maladie Xundheit écrit: «notre médecin-conseil ne prendra position sur l'obligation de verser des prestations dans le cas de cette facture [...] qu'après avoir reçu les rapports médicaux originaux demandés.» (trad. FMH) La caisse-maladie Xundheit donne l'adresse directe du médecin-conseil indiqué nommément.

Le 16 janvier 2004, le médecin traitant s'adresse en ces termes au médecin-conseil: «[...] je te communique que je suis entièrement prêt à te livrer les données nécessaires pour l'accomplissement de ta tâche. En tant que médecin, je suis toutefois aussi tenu à te livrer uniquement les données nécessaires à la détermination des prestations qui doivent être versées par la caissemaladie [...]. Par conséquent, communiquemoi ce que tu veux et dois savoir de moi.» (trad. $\mathrm{FMH})$
Le 23 janvier 2004, le médecin-conseil répond au médecin traitant par une lettre qu'il signe personnellement: «Effectivement, il ne s'agit pas ici, pour moi, de contrôler le traitement mais de vérifier, à la demande de la caisse, si la facturation des rapports médicaux contestés est correcte. Je ne peux le faire, bien entendu, qu'en consultant ces rapports et non pas en posant des questions quelconques.» (trad. FMH)

A la demande de l'auteur, le médecin-conseil concerné complète aujourd'hui les antécédents du cas d'espèce en ces termes [1]: le médecin en question «était, depuis longtemps, très bien connu des caisses-maladie et médecins-conseils et ses factures ont été régulièrement soumises à des contrôles» (trad. FMH). Des années auparavant, «sa manière de travailler et de facturer a fait l'objet d'une procédure de la CPC qui s'est malheureusement achevée par un non-lieu parce que les représentants du corps médical n'ont pas eu le courage, à l'époque, d'en accepter les mesures.» (trad. FMH) Le médecin-conseil indique encore ceci: «Cinq rapports médicaux partiellement volumineux établis en quatre jours sont suspects. Je voulais vérifier leur contenu et les destinataires de rapports d'une telle ampleur. Pour cette raison, je tenais à consulter ces rapports et ne pouvais pas poser de questions.» (trad. FMH)

\section{Commentaire concernant les antécédents} Si le médecin traitant nous avait contactés le 23 janvier 2004, nous lui aurions recommandé d'envoyer les rapports concernés au médecinconseil.

\section{Le jugement du tribunal cantonal des assurances}

Les rapports n'ont été envoyés au médecinconseil ni par le médecin traitant ni par la patiente. Celle-ci présenta l'affaire devant le tribu- 
nal cantonal des assurances suite à la décision formelle et à la décision sur opposition de la caisse. Selon elle, le médecin traitant est tenu au secret médical et qu'en sa qualité de médecin actif en gynécologie, il ne peut remettre au médecin-conseil d'une caisse-maladie aucune lettre adressée à l'hôpital ou un autre spécialiste [2]

Le président du tribunal cantonal des assurances répertoria les traitements ainsi que les cinq rapports médicaux facturés pour le mois de septembre 2002 et releva ce qui suit [3]: «D'une part, il est compréhensible que le médecinconseil veuille vérifier pour quelle raison il a été nécessaire de rédiger autant de rapports. D'autre part, il y a aussi un intérêt à savoir si ces rapports peuvent avoir un lien avec d'autres diagnostics ou traitements qui n'étaient éventuellement pas des prestations obligatoires. Après qu'une position 〈rapport intermédiaire, certificat médical〉a été à nouveau facturée le 10 novembre 2003, il est donc juste que le médecin-conseil veuille voir, outre ce nouveau rapport, également les premiers rapports pour pouvoir conseiller correctement la partie intimée [la caisse-maladie; remarque de l'auteur].» (trad. FMH).

\section{Commentaire concernant le jugement cantonal}

Vu le cas concret, il apparaît que le médecinconseil doit pouvoir vérifier la nécessité à la base de l'accumulation de rapports et que, pour ce faire, il a besoin des rapports eux-mêmes. En revanche, le deuxième argument du Tribunal contredit les motifs indiqués le 23 janvier 2004 par le médecin-conseil au médecin traitant. Cet argument est pour le moins formulé de manière à créer des malentendus, s'il n'est pas carrément faux du point de vue logique: si les rapports à d'autres médecins ont effectivement concerné des «examens et traitements qui n'étaient éventuellement pas des prestations obligatoires», ces passages devraient, à mon avis, être couverts dans la copie transmise au médecin-conseil. En effet, si je paye déjà moi-même un traitement, celui-ci ne regarde alors pas l'assurance sociale.

\section{Contrôle par sondages des rapports médicaux par le médecin-conseil: légitime sur le fond}

Dans le cours de formation postgraduée en vue de l'attestation de formation complémentaire «médecin-conseil», nous avons entendu et entendons encore de différents médecins-conseils que ces dernières années, il y a quand même eu un nombre importants de rapports médicaux facturés de façon incorrecte. De ce fait, j'estime légitime qu'un médecin-conseil contrôle par sondage des rapports médicaux. Je cite parmi nos exemples d'exercices réalisés en commun en 2006 [4] pour le cours de formation postgraduée en vue de l'attestation «médecin-conseil»:

«19. Votre caisse vous demande de contrôler, en tant que médecin-conseil, si les rapports médicaux sont facturés correctement: vous devez concrètement compter le nombre de lignes. Que faites-vous? Un contrôle par sondage consistant à ‘compter les lignes> peut être justifié - un contrôle régulier pour tous les fournisseurs de prestations ne le serait pas. En règle générale [5], le médecin-conseil transmet de manière judicieuse le comptage des lignes effectué dans le cadre d'un contrôle par sondage à sa secrétaire, laquelle travaille selon les directives qu'il lui donne.

19.1. Variante: trois mois après, la caisse veut savoir si la patiente présente une grossesse accompagnée de symptômes morbides ( $\rightarrow$ participation aux coûts de l'assurée). Votre secrétaire se souvient que la réponse serait à trouver dans le rapport dont elle a compté les lignes pour vous. Question: Quel renseignement donnez-vous à la caisse?

Les rapports demandés dans le cadre de l'action Compter les lignes ne sont pas déposés dans le dossier de l'assuré se trouvant chez le médecin-conseil. Le rapport doit être demandé une nouvelle fois.

(Raison: la caisse doit déclarer explicitement l'action aux médecins traitants dès le début, y compris le but indiqué. Respecter le but indiqué signifie compter seulement les lignes, détruire les rapports facturés correctement et archiver les rapports facturés de manière incorrecte en vue d'une procédure d'opposition, mais pas dans le dossier de l'assuré.)»(trad. FMH)

\section{Commentaire sur l'arrêt du TFA \\ du 18 mai 2006}

Pour le résumé de l'arrêt proprement dit, je renvoie à l'article même de Christian Peter.

1. L'arrêt a été prononcé par un tribunal composé de trois juges et non de cinq. Pour des raisons formelles déjà, il n'est pas un arrêt fondamental.

2. Dans cet arrêt, le TFA se base sur l'idée que les médecins-conseils des assureurs-maladie accomplissent leur fonction légale de filtre de manière fiable. Reste à savoir si c'est réellement le cas chez toutes les caisses. Le service juridique de la FMH entend régulièrement des médecins traitants dire que la réponse qui leur est parvenue à une lettre envoyée au médecinconseil émane du département des prestations (et non du médecin-conseil), lettre qui laisse, par son contenu profane, impérativement supposer que le médecin-conseil n'a pas du tout reçu le courrier qui lui était adressé. Les discussions informelles tenues dans le cours de formation postgraduée pour l'attestation de médecin-conseil ne sont pas non plus toujours rassurantes lorsqu'elles portent sur ce point. Les assureurs-maladie et les médecins-conseils, ainsi que l'OFSP à titre d'autorité de surveillance, assument la respon- 
sabilité quant à la garantie et à l'application de processus corrects.

3. En l'espèce, j'estime que le TFA s'est prononcé correctement dans les conclusions: le médecin traitant aurait dû remettre les rapports médicaux au médecin-conseil après la demande personnelle et dûment fondée de ce dernier du 23 janvier 2004. Je pense que tous les patients, médecins, médecins-conseils et assureurs peuvent soutenir cette conclusion.

4. Par contre, les motifs me semblent incompréhensibles. A mon avis, le TFA aurait pu et dû, dans son arrêt, se limiter aux considérations suivantes: Le médecin-conseil a voulu, en l'occurrence, «vérifier si la facturation des rapports contestés était correcte» (trad. FMH) (et non pas le traitement proprement dit); cette demande étant admissible, il fallait lui remettre lesdits rapports; par voie de conséquence, les rapports médicaux remis après un contrôle positif ne doivent pas être archivés dans le dossier de l'assuré se trouvant chez le médecin-conseil (Principes - Art. 4, 3e al. de la loi sur la protection des données: «Les données personnelles ne doivent être traitées que dans le but qui est indiqué lors de leur collecte, qui est prévu par une loi ou qui ressort des circonstances.» S'il ne s'agit pas du traitement mais uniquement des rapports, ceux-ci n'ont pas leur place dans le dossier des patients.)

Le fait que la lettre, décisive à mon avis, envoyée le 23 janvier 2004 au médecin par le médecinconseil, ne soit pas mentionnée dans les arrêts des deux instances, est une lacune difficilement compréhensible - cette correspondance n'étaitpas connue du tribunal? Eu égard à l'histoire concrète du cas, les considérants 4 et 5 du TFA au sujet du droit général de la caisse de demander des rapports au médecin-conseil apparaissent dans de longs passages comme simples obiter dicta; «Comme ils vont au-delà de la tâche du tribunal de juger le litige concret, ces considérants ne sont pas sans poser de problèmes et il faut les considérer avec prudence.» [6] (trad. FMH).

\section{Recommandations}

Je partage la position de Christian Peter en complétant un troisième point:

1. En cas d'éléments obscurs, le département des prestations de la caisse-maladie concernée devrait poser des questions au médecin traitant.

2. Il revient au médecin-conseil - et non à l'administration de la caisse - de décider et de justifier quand et pourquoi il a besoin d'une copie d'un rapport médical. En l'espèce, le médecin-conseil a agi de manière exemplaire.

3. Les directeurs des caisses-maladie et les médecin-conseils doivent garantir la réception, par les médecins-conseils, du courrier qui leur est adressé, ainsi que l'exige la loi. L'Office fédéral de la santé publique devrait le contrôler par sondage parce que la personne obligatoirement assurée pour la maladie doit pouvoir compter sur un fonctionnement des caissesmaladie selon la loi.

\section{Références}

1 Réponse à l'auteur par courriel du $1^{\text {er }}$ février 2007.

2 Selon jugement du tribunal cantonal des assurances du 13 décembre 2004, p. 2.

3 Jugement du tribunal canton des assurances, E. 2.a.

4 Corinne Zbären-Lutz, Hanspeter Kuhn, Kurt Schweingruber - exercices concernant la protection des données [pour la formation postgraduée en vue de l'attestation de médecin-conseil], octobre 2006.

5 Complément janvier 2007.

6 Voir pour Obiter Dicta (latin: propos secondaire) chez Peter Forstmoser et Regina Ogorek, Juristisches Arbeiten, Eine Anleitung für Studierende,

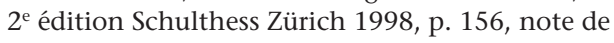
bas de page 86: «A l'occasion, certains tribunaux saisissent l'occasion présentée par la justification d'un arrêt pour émettre des considérations qui ne sont pas nécessaires pour l'arrêt dans le cas concret. On part alors de obiter dicta ou de dicta. Comme ils vont au-delà de la tâche du tribunal de juger le litige concret, ces considérants ne sont pas sans poser de problèmes et il faut les considérer avec prudence.» 